\title{
Holography, 1-form symmetries, and confinement
}

\author{
Fabio Apruzzi, Marieke van Beest๑, Dewi S. W. Gould@, and Sakura Schäfer-Nameki॰ \\ Mathematical Institute, University of Oxford, Woodstock Road, Oxford OX2 6GG, United Kingdom
}

(Received 3 May 2021; accepted 1 August 2021; published 2 September 2021)

\begin{abstract}
We study confinement in $4 \mathrm{~d} \mathcal{N}=1 S U(N)$ Super-Yang Mills (SYM) from a holographic point of view, focusing on the 1-form symmetry and its relation to chiral symmetry breaking $\left(\chi_{\mathrm{SB}}\right)$. We identify the topological couplings in the $5 \mathrm{~d}$ truncation of the Klebanov-Strassler solution that determine the 1-form symmetry and its 't Hooft anomalies. One coupling is a mixed 0 -/1-form symmetry anomaly related to $\chi_{\mathrm{SB}}$ in gapped confining vacua. In the gravity dual we also identify the infrared $4 \mathrm{~d}$ topological field theory which realizes $\chi_{\mathrm{SB}}$ and matches the mixed anomaly. Finally, complementing this, we derive the chiral and mixed anomalies from the little string theory realization of pure SYM.
\end{abstract}

DOI: 10.1103/PhysRevD.104.066005

\section{INTRODUCTION}

Generalized global symmetries and their 't Hooft anomalies can highly constrain the dynamics of gauge theories. A prime example is the role of 1-form symmetries in confinement of $\mathcal{N}=1 S U(N)$ super Yang-Mills (SYM) or adjoint QCD theories. In this case the 1-form symmetry $\Gamma^{(1)}=\mathbb{Z}_{N}$ and corresponds to the center of the gauge group, which acts on line operators [1,2] and provides a diagnostic of confinement. The order parameter for this symmetry is the vacuum expectation value (vev) of the Wilson line in the fundamental representation, which obeys area law in a confining vacuum. This implies vanishing of the vev of an infinitely extended Wilson line, thus preserving the 1-form symmetry. In addition, $\mathcal{N}=1 S U(N)$ SYM also has a 0 form R symmetry $U(1)_{R}^{(0)}$. The Adler-Bell-Jackiw (ABJ) or chiral anomaly breaks $U(1)_{R}^{(0)}$ to $\Gamma^{(0)}=\mathbb{Z}_{2 N}$, which by $\chi_{\mathrm{SB}}$ $[3]$ reduces to $\mathbb{Z}_{2}$ in the confining phase

$$
U(1) \stackrel{\mathrm{ABJ}}{\longrightarrow} \mathbb{Z}_{2 N} \stackrel{\chi_{\mathrm{SB}}}{\longrightarrow} \mathbb{Z}_{2}
$$

There is a 0-/1-form symmetry mixed 't Hooft anomaly,

$$
\mathcal{A}\left[b_{2}, A\right]=2 \pi N^{2} \int_{X_{5}} A b_{2} b_{2}
$$

where $b_{2}$ is the background for $\mathbb{Z}_{N}^{(1)}$ and $A$ for $\Gamma^{(0)}$, which satisfy $\oint b_{2} \in \frac{\mathbb{Z}}{N}$ and $\oint A \in \frac{\mathbb{Z}}{2 N}$. This anomaly constrains the

Published by the American Physical Society under the terms of the Creative Commons Attribution 4.0 International license. Further distribution of this work must maintain attribution to the author(s) and the published article's title, journal citation, and DOI. Funded by SCOAP ${ }^{3}$. infrared (IR) strongly coupled physics $[2,4,5]$. In a confining vacuum the 1 -form symmetry is unbroken. The 0 -form background has to satisfy $\oint A \in \frac{\mathbb{Z}}{2}$, and $\Gamma^{(0)}$ is broken to $\Gamma^{(0)}=\mathbb{Z}_{2}$. This breaking indicates $N$ distinct confining vacua, modeled by a gapped topological field theory (TQFT).

The goal of this paper is to develop new methods to compute anomalies of discrete higher-form symmetries and moreover provide a derivation of the TQFT sectors, which match these anomalies, without relying on anomaly matching or a Lagrangian description of the UV. This paper provides a proof of concept by successfully implementing this in the context of the confinement of $4 \mathrm{~d} \mathcal{N}=1 \mathrm{SYM}$. What we show is that the dual supergravity theory captures not only the background fields for the higher-form symmetries, and their anomalies, but also provides a derivation of the $4 \mathrm{~d}$ TQFT that governs the IR confining phase of the theory.

Higher-form symmetries in the AdS/CFT correspondence were discussed in [6-11]. Our focus here is on holography in a nonconformal setting, providing a dual to $\mathcal{N}=1 S U(N)$ SYM [12-15]. Concretely, we determine the 1-form symmetry and its anomalies in the KlebanovStrassler (KS) [16-21] solution and derive the theory in the far IR. The central tool for our analysis is the consistent truncation of supergravity to $5 \mathrm{~d}$ [22-24].

The main results of this paper are as follows: From the $5 \mathrm{~d}$ topological couplings, we identify the 1-form symmetry and anomalies that are central to $\chi_{\mathrm{SB}}$. Perhaps even more strikingly, we derive the TQFT description of the gapped IR confining vacua of $\mathcal{N}=1 S U(N)$ SYM. We show how the $5 \mathrm{~d}$ supergravity reduction remarkably contains the Lagrangian of the $4 \mathrm{~d}$ TQFT describing the IR physics, providing an independent derivation of the existence of this TQFT sector, which agrees with but does not rely on anomaly matching. Our result for $\mathcal{N}=1 S U(N)$ SYM 
provides an exposition of a more general methodology: utilizing supergravity couplings to determine anomalies and IR sectors of quantum field theories (QFTs). There are a vast number of applications where the topological couplings are determined at the boundary of the compactification space, from holography to geometric engineering of QFTs in string theory, e.g., [25], but also in setups with broken supersymmetry (where the KS solution plays a key role). We expect our work to open up the exciting prospect of revisiting these setups and sharpening their predictions and extending the scope of such scenarios.

\section{HOLOGRAPHIC DUAL TO CONFINEMENT}

One of the most successful holographic realizations of $\mathcal{N}=1 S U(N)$ SYM theory is the KS solution [17]. This construction is realized in 10d IIB supergravity, and it consists of two main ingredients: (1) $N$ D3-branes probing the conifold $C\left(T^{1,1}\right)$, which is a conical Calabi-Yau with $5 \mathrm{~d}$ link $T^{1,1}$, that is topologically $S^{2} \times S^{3}$. The near-horizon of this brane system is $\mathrm{AdS}_{5} \times T^{1,1}$ with 5-form flux $\int_{T^{1,1}} F_{5}=N$. (2) $M$ D5-branes wrapping the $S^{2} \subset T^{1,1}$. The D5s backreact on the external geometry, modifying the $\mathrm{AdS}_{5}$ metric. The KS solution at large $r$ is

$\mathrm{d} s_{10}^{2}=\mathrm{d} s_{\mathcal{M}_{5}}^{2}+\mathcal{R}^{2}(r) \mathrm{d} s_{T^{1,1}}^{2}, \quad \mathcal{R}(r) \sim \ln \left(r / r_{s}\right)^{1 / 4}$,

where $\mathrm{d} s_{\mathcal{M}_{5}}^{2}=\frac{r^{2} \mathrm{~d} \vec{x}^{2}}{\mathcal{R}^{2}(r)}+\frac{\mathcal{R}^{2}(r) \mathrm{d} r^{2}}{r^{2}}$ and $r_{s}=r_{0} e^{-\frac{2 \pi N}{3 g_{s} M^{2}}-\frac{1}{4}}$. Here $r_{0}$ is the UV scale, and we refer to this as the UV KS solution, valid for $r$ sufficiently large and $g_{s} \mathcal{K}(r) \gg 1$. At large $r$, the quantization of fluxes is

$$
\begin{aligned}
\int_{S^{3}} F_{3} & =M, \quad \int_{S^{2}} B_{2}=\mathcal{L}(r), \\
\int_{T^{1,1}} F_{5} & =\mathcal{K}(r)=N+M \mathcal{L}, \quad \mathcal{L}=\frac{3 g_{s} M}{2 \pi} \ln \left(r / r_{0}\right) .
\end{aligned}
$$

Note that $F_{5}$ is no longer quantized: its integral over the internal space acquires a radial dependence. The solution has a naked singularity at $\mathcal{R}\left(r_{s}\right)=0$, and in particular, we can consider $r_{s} \rightarrow 0$, when $\frac{N}{M^{2}} \gg 1$. Therefore, at small radial distances $r \rightarrow r_{s}$, (3) is no longer valid but gets replaced by the full warped, deformed conifold solution [17], as summarized in [26].

The dual field theory description is given by $S U(N+$ $M) \times S U(N)$ gauge theory and bifundamental matter in $(\mathbf{N}+\mathbf{M}, \overline{\mathbf{N}}) \oplus(\overline{\mathbf{N}}+\mathbf{M}, \mathbf{N})$, where a combination of the two gauge couplings flows strong coupling. In particular, this theory is not conformal, and the gauge couplings of the two factors run in opposite directions. For example, when $S U(N+M)$ with $N_{F}=2 N$ becomes strongly coupled, we apply Seiberg duality [27], resulting in $S U(N-M)$ with $N_{F}=2 N$. This process perpetuates with the new gauge couplings flowing in opposite directions, giving rise to a "duality cascade". For $N=\kappa M, \kappa \in \mathbb{N}$, the end point is $\mathcal{N}=1 S U(M)$ SYM at strong coupling.

The RG flow of the gauge theory cascade is mirrored explicitly in the dual gravity background. Moving from large $r$ to $r \rightarrow r e^{-\frac{2 \pi}{3 g_{s} M}}, \int_{S^{2}} B_{2}$ and $\int_{T^{1,1}} F_{5}$ change by $\mathcal{L}(r) \rightarrow \mathcal{L}(r)-1, \mathcal{K}(r) \rightarrow \mathcal{K}(r)-M$. At slices $r=r_{k}=$ $r_{0} e^{-\frac{2 \pi k}{3 g_{s} M}}$, where $\mathcal{L}, \mathcal{K}$ are integers, the supergravity background is dual to the $S U(N-(k-1) M) \times S U(N-k M)$ gauge theory in the baryonic branch [28]. Alternatively we can work in terms of Page charges defined in [29], where $\hat{F}_{5}=F_{5}-B_{2} F_{3}$ is always integrally quantized $\int_{T^{1,1}} \hat{F}_{5}=N-k M$, due to large gauge transformations of $\int_{S^{2}} B_{2} \rightarrow \mathcal{L}(r)+k$. For $N=\kappa M$, the end point is reached at a value $r_{\kappa}$ where there are only $M$ units of $F_{3}$-flux and no $F_{5}$-flux. In this regime, $r \sim r_{k}$, the solution (3) breaks down before reaching the $r \rightarrow r_{s}$ limit, since $g_{s} \mathcal{K}\left(r_{\kappa}\right)=0$, and the metric in (3) is not smooth. We therefore have the following hierarchy of scales: $r_{0} \gg r_{k} \gg r_{\kappa}>r_{s}$.

\section{1-FORM SYMMETRIES FROM SUPERGRAVITY}

The global form of the gauge group, or put differently, the set of mutually local line operators, can be determined in holography by considering boundary conditions (b.c.) of Chern-Simons-like couplings [6-8]. Put in a more modern language, the 2-form backgrounds for 1-form symmetries of the holographic field theory are determined by topological couplings in the bulk, and specific b.c.s yield absolute theories (i.e., definite spectra of line operators). On the gauge theory side of the duality cascade, the $\mathbb{Z}_{N+M} \times \mathbb{Z}_{N}$ center symmetry of $S U(N+M) \times S U(N)$ is broken by the matter to

$$
\Gamma^{(1)}=\mathbb{Z}_{\operatorname{gcd}(N, N+M)}=\mathbb{Z}_{\operatorname{gcd}(N, M)} .
$$

This remains invariant along the $\mathrm{RG}$ flow.

In order to derive the 1-form symmetry holographically we study fluctuations around the UV KS solution, when $r$ is sufficiently large and $g_{s} \mathcal{K}(r) \gg 1$, which describes each step of the cascade until $r \sim r_{\kappa}$. The latter corresponds to the end of the cascade, and the smooth gravity dual is the warped deformed conifold, which we investigate momentarily. We reduce IIB supergravity on $T^{1,1}$ and study the topological couplings of 2 -form gauge fields. The strings which couple to these fields induce line operators on the $r_{k}$ slices, which in turn furnish the 1-form symmetry of the boundary theory, also known as a "singleton theory" [30,31]. In particular, the 1-form symmetry is deduced from the bulk couplings by imposing a set of consistent b.c.s in which a subset of the 2-form gauge potentials are fixed in a subgroup of $U(1)$. We first consider fluctuations of the fluxes around the background on the cohomology of $T^{1,1}$, i.e., $F_{q}$ expanded along $\omega_{p} \in H^{p}\left(T^{1,1}, \mathbb{Z}\right)$ as 
$F_{q}=\sum_{p} f_{q-p} \wedge \omega_{p}$. In this case the nontrivial cohomology elements are $\omega_{2}$ and $\omega_{3}$, i.e., the volume forms of $S^{2}$ and $S^{3}$, respectively. The fluctuations of the IIB fluxes around the KS solution read

$$
\begin{aligned}
& \delta H_{3}=d b_{2}, \quad \delta F_{3}=d c_{2}+\omega_{2} \wedge d c_{0}, \\
& \delta F_{5}=\omega_{2} \wedge f_{3}+\omega_{3} \wedge * f_{3},
\end{aligned}
$$

where these expansions already satisfy the Bianchi identities for $H_{3}, F_{3}, F_{5}$ and self-duality $F_{5}=* F_{5}$ (see [26]). Here, $c_{0}$ is a $2 \pi$ periodic scalar, whereas $b_{2}, c_{2}$ couple to F1s and D1s, respectively. We use $f_{3}$, since the operators of the boundary 1-form symmetry are manifest in this frame. The Bianchi identity for $F_{5}$ sets $f_{3}-\mathcal{L} d c_{2}-b_{2} d c_{0}=d a_{2}$, which introduces a 2-form gauge field sourced by D3s wrapping the $S^{2} \subset T^{1,1}$.

In order to get the $5 \mathrm{~d}$ bulk topological action describing the singleton theory, we implement the following strategy. We reduce the flux equations of motion of IIB supergravity on $T^{1,1}$ and construct a classical $5 \mathrm{~d}$ action which realizes these equations of motion. We study this at $r=r_{k}+r^{\prime}$, $r^{\prime} \ll r_{k}$, which has a field theory dual. For $k \ll \frac{N}{M}$ we are far away from the IR cutoff, and in this regime the topological terms dominate. The effective $F_{5}$ charge is $\mathcal{K}(r)=$ $N-k M+\mathcal{O}\left(r^{\prime} / r_{k}\right)$, so the leading contributions to the equations of motion are the topological couplings

$$
\operatorname{gcd}(N, M) d \mathcal{C}=0, \quad \operatorname{gcd}(N, M) d b_{2}=0,
$$

where $\mathcal{C}=q_{1} c_{2}-q_{2} a_{2}$, with $\operatorname{gcd}(N, M) q_{1}=N, \operatorname{gcd}(N$, $M) q_{2}=M$, and whereby we decoupled the center of mass $U(1)^{(1)}$, corresponding to the 1 -form symmetry of the collective motion of the D3s. The couplings are embedded into the consistent truncation of [22]. One can compare by varying their topological action, changing the duality frame and restricting to the relevant fields. We find that the following topological term in the $5 \mathrm{~d}$ supergravity reduction on the UV KS solution at $r=r_{k} \gg r_{k}$ dominates over higher derivative couplings,

$$
S_{5 \mathrm{~d}} \supset 2 \pi \operatorname{gcd}(N, M) \int_{\mathcal{M}_{5}} b_{2} \wedge d \mathcal{C} .
$$

1-Form symmetries are generated by topological surface operators [2], which are realized by the bulk operators $U_{b}\left(M_{2}\right)=e^{2 \pi i \oint_{M_{2}} b_{2}}$ and $U_{c}\left(M_{2}\right)=e^{2 \pi i \oint_{M_{2}}{ }^{C}}$, where $M_{2}$ are closed surfaces, $\partial M_{2}=\emptyset$. Generically, due to noncommutativity of fluxes, these do not commute [6],

$$
U_{b}\left(M_{2}\right) U_{c}\left(N_{2}\right)=U_{c}\left(N_{2}\right) U_{b}\left(M_{2}\right) e^{\frac{2 \pi i L\left(M_{2}, N_{2}\right)}{N}},
$$

where $L$ is the linking of the surfaces. These charge operators generate a 1-form symmetry, which acts on charged line operators in the $4 \mathrm{~d}$ field theory. For each slice $\left.\mathcal{M}_{5}\right|_{r_{k}}$ ((3) evaluated at $r=r_{k}$ ) charged line operators are $U_{b}(\Sigma)=e^{2 \pi i \oint_{\Sigma} b_{2}}, U_{c}(\Sigma)=e^{2 \pi i \oint_{\Sigma} \mathcal{C}}$ with $\left.\partial \Sigma \subset \mathcal{M}_{5}\right|_{r_{k}}$ [32]; however only a maximal set of mutually local line operators are allowed, which are encoded in the b.c.s of $b_{2}$ and $\mathcal{C}$.

A possible choice of b.c. for (8) is $b_{2}$ Dirichlet and $\mathcal{C}$ Neumann. Since $\mathcal{C}$ is free to vary at the boundary, $U_{c}$ will correspond to the topological charge operator for the 1 -form symmetry. By varying the topological action we find a condition $\left.\operatorname{gcd}(N, M) b_{2} \wedge \delta \mathcal{C}\right|_{r_{k}}=0$, which forces $b_{2}$ to take fixed values at the boundary. This implies that we can define a flat connection $b_{1}$ in $4 \mathrm{~d}$ taking values in $\mathbb{Z}_{\operatorname{gcd}(N, M)}$, i.e., $\operatorname{gcd}(N, M) b_{2}=d b_{1}=0$ at the slice $r=r_{k}$. Therefore, $U_{b}$ restricted to $\left.\partial \Sigma \subset \mathcal{M}_{5}\right|_{r_{k}}$ corresponds to the charged line operators of the field theory. As is well known, the fundamental strings, carrying world-volume $b_{2}$, ending on the boundary indeed give rise to Wilson lines in the $4 \mathrm{~d}$ theory. They generate the 1-form symmetry (5).

The screening can equally be seen by considering the analog of the "baryon vertex" [33] in this setup: integrating the Bianchi identities for D5s on $T^{1,1}$ and D3s on $S^{3}$ yields

$$
\begin{aligned}
\int_{T^{1,1}} d F_{7} & =\int_{T^{1,1}} H_{3} \wedge F_{5}=(N-k M) H_{3}, \\
\int_{S^{3}} d F_{5} & =\int_{S^{3}} H_{3} \wedge F_{3}=M H_{3} .
\end{aligned}
$$

Thus D5s on $T^{1,1}$ screen $N-k M$ F1s, and D3s on $S^{3}$ screen $M$ F1s. The minimal configuration of screened strings is thus $\operatorname{gcd}(N, M)$. Alternative b.c.s are detailed in [26]. From here onwards, $N=\kappa M$, where the bottom of the cascade is a confining phase $S U(M)$ SYM. The b.c.s are $M d b_{2}=0$, i.e., F1 strings ending on the boundary. This is detailed in [26], and we see how to derive this condition from the $5 \mathrm{~d}$ bulk topological action momentarily.

\section{MIXED ANOMALY AND $\chi_{\text {SB }}$ FROM HOLOGRAPHY}

All things are now in place to derive holographically the mixed anomaly (2). To do this, we need to study the rest of the topological couplings in the $5 \mathrm{~d}$ bulk supergravity action. In particular, we need to include the $\mathrm{R}$ symmetry of the dual field theory, which is realized in terms of the $U(1)$ isometry (Reeb-vector) of the $T^{1,1}$ solution. This can be described by a $U(1) 1$-form gauge field $A$, which enters the metric of $T^{1,1}$ as $\mathrm{d} \beta \rightarrow \mathrm{d} \beta-A$, where $\beta$ is the coordinate of the Hopf fiber of the $S^{3}$. The breaking by the ABJ anomaly to $\mathbb{Z}_{2 M}$ is realized holographically by a Stückelberg coupling in the $5 \mathrm{~d}$ consistent truncation. We also argue that $\chi_{\mathrm{SB}}$ is consistent with the mixed 0-/1-form anomaly, which we derive from the $5 \mathrm{~d}$ supergravity. Perhaps most strikingly, we derive the TQFT that governs 
the IR from first principles, confirming the anomaly-based arguments in [2].

The additional $5 \mathrm{~d}$ topological terms in the action are

$S_{5 \mathrm{~d}} \supset 2 \pi \int \frac{\mathcal{R}}{2}\left|d c_{0}+2 M A\right|^{2}+M^{2} b_{2}^{2} A+\frac{M}{2} b_{2}^{2} d c_{0}$.

The first term is the kinetic term for the axion. Since it contains two derivatives it is subleading when evaluated on the UV KS solution, when $r$ is large, with respect to the topological terms. On the other hand its effect is important, since it realizes the Stuickelberg mechanism for the $U(1)_{R}$ gauge field $A$. The shift symmetry of the axion, $c_{0} \sim c_{0}+2 \pi$, is gauged by the $U(1)_{R}$ symmetry, so that the action is invariant under the nonlinear transformation

$$
A \rightarrow A+\mathrm{d} \alpha, \quad c_{0} \rightarrow c_{0}-2 M \alpha .
$$

We can use this symmetry to completely gauge away the axion, leaving only a mass term for the gauge field. Fixing $c_{0}=0$, there is still a residual discrete symmetry generated by $\alpha \in \frac{\pi}{M} \mathbb{Z}$. This is the direct way to identify the breaking of $U(1)_{R} \rightarrow \mathbb{Z}_{2 M}^{(0)}$, as required by the ABJ anomaly.

The second term in (11) corresponds to the anomaly between the 0 -form background $A$ for $\mathbb{Z}_{2 M}^{(0)}, \oint A \in \frac{\mathbb{Z}}{2 M}$, and $b_{2}$ for $\mathbb{Z}_{M}^{(1)}, \oint b_{2} \in \frac{\mathbb{Z}}{M}$

$$
\mathcal{A}\left[b_{2}, A\right]=+2 \pi M^{2} \int_{\mathcal{M}_{5}} b_{2} b_{2} A,
$$

which is a mixed 0-/1-form symmetry anomaly [34]. As expected it does not depend on the energy scale, and therefore this term will survive in the IR. In the IR we expect the theory to be dual to a confining vacuum of $S U(M)$ SYM, so the $\mathbb{Z}_{M}^{(1)}$ should be unbroken, and this gapped phase should be described by a $4 \mathrm{~d}$ TQFT. Assuming $\mathcal{M}_{5}$ is spin, then $b_{2}^{2}$ is even. Since $A$ is a $\mathbb{Z}_{2 M}$ background, (13) is not integral, in general. It was proven in [5] that unless this term is integral there cannot be a $4 d$ TQFT with $\Gamma^{(0)}=\mathbb{Z}_{2 M}$ and $\Gamma^{(1)}=\mathbb{Z}_{M}$ symmetries in the IR that saturates all the anomalies of the theory in the UV. On the other hand, integrality of (13) and an unbroken $\mathbb{Z}_{M}^{(1)}$ implies $\oint A \in \frac{\mathbb{Z}}{2}$, implying $\chi_{\mathrm{SB}}$ in the IR vacuum of $S U(M)$ SYM. We stress that our analysis shows that the presence of this topological coupling in the UV KS supergravity solution is already preempting and consistent with the chiral symmetry breaking in the gapped confining vacuum with $\mathbb{Z}_{2}^{(0)}$ and $\mathbb{Z}_{M}^{(1)}$ symmetries.

\section{4D IR TQFT FROM HOLOGRAPHY}

Finally, we now turn to the derivation of the IR description of the confining vacua. From the 5d supergravity topological terms we derive the action of the IR $4 \mathrm{~d}$
TQFT, which matches (13), and realizes spontaneous chiral symmetry breaking, $\mathbb{Z}_{2 M} \rightarrow \mathbb{Z}_{2}$. Based on a field theoretic anomaly matching argument, a candidate TQFT was proposed in [2]

$$
S_{\mathrm{TQFT}_{4 \mathrm{~d}}}=\int M \phi\left(d c_{3}+\frac{M}{2} b_{2}^{2}\right)=\int M \phi F_{4} .
$$

The $M$ vacua, labeled by $\left\langle e^{i \phi}\right\rangle=e^{\frac{2 \pi i \ell}{M}}, \ell=0,1, \ldots M-1$, are separated by domain walls (DWs) [35], $e^{i \oint c_{3}}$. We now give a holographic derivation of the IR TQFT based on the $5 \mathrm{~d}$ bulk couplings of the IR KS solution.

The smooth IR gravity dual background is the deformed conifold solution, where $\tau \rightarrow 0$ (see [26]). In this regime there is no hierarchy between the $5 \mathrm{~d}$ bulk kinetic and topological terms, and the former need to be taken into account. Before the $S^{2}$ degenerates, the D5s source $C_{6}=\omega_{3} \wedge c_{3}$, and in addition since $F_{7}=* F_{3}$ we consider $d c_{3}=\frac{\mathcal{R}}{2} *_{5}\left(d c_{0}+2 M A\right)$. Therefore, in the IR the dynamics of $c_{0}$ becomes relevant. Since $A$ corresponds to the true isometry of the IR KS solution, i.e., is a $\mathbb{Z}_{2}$ gauge field, $c_{0}$ does not shift under a gauge transformation of $A$. The $5 \mathrm{~d}$ IR topological action becomes

$$
S_{5 \mathrm{~d}}^{\prime} \supset 2 \pi \int 2 M A d c_{3}+d c_{0} d c_{3}+\frac{M}{2} b_{2}^{2} d c_{0} .
$$

We first notice that the mixed anomaly between $\Gamma^{(0)}=\mathbb{Z}_{2 M}$ and $\Gamma^{(1)}=\mathbb{Z}_{M}$ has disappeared. This is due to an additional topological term $\left|c^{\Omega}(r)\right|^{2} b_{2}^{2} A$ [22,36,37], which for the UV KS solution depends on the UV scale $r_{0}$, but is constant in the IR $c^{\Omega}=M$. This is consistent with anomaly matching since the IR theory has $\Gamma^{(0)}=\mathbb{Z}_{2}$, which is not anomalous on spin manifolds. The third term is a total derivative, and varying by $c_{0}$ implies $M d b_{2}=0$, in agreement with the equations of motion on the deformed conifold. When this condition is satisfied, the last two terms give rise to topological counterterms for the $4 \mathrm{~d}$ theory living at the boundary. This implies that they are not anomalies, but rather the imprint of the TQFT (14) in the IR, which is precisely obtained by identifying $c_{0} \leftrightarrow M \phi$ and evaluating these terms at the boundary. In particular, $c_{0}$ is related to the presence of DWs given by D5s wrapping $S^{3}$. These source $\int_{B} F_{3}$, where $B$ is the Poincare dual cycle in the deformed conifold with $S^{2}$ boundary at infinity. This entails that $\int_{B} F_{3} \sim \int_{S^{2}} c_{0} \omega_{2}$, and because of the presence of the D5 DWs, $c_{0}=\ell$ is quantized and corresponds to the number of D5s. The UV anomaly (13) in the IR is realized by the action of $\Gamma^{(0)}=\mathbb{Z}_{2 M}, \ell \rightarrow \ell+1$, which is however not a symmetry of the IR vacuum.

We emphasize that this derivation did not make use of any anomaly matching arguments, and thereby provides a top-down derivation of the theory governing the IR! 
This IR theory is furthermore invariant under 1-form symmetry transformations $b_{2} \rightarrow b_{2}+d \lambda$, which implies that $c_{3} \rightarrow c_{3}-M b_{2} \lambda-\frac{M}{2} \lambda d \lambda$. The fact that the transformation of the 1-form symmetry enters in the shift of $c_{3}$ signals the presence of a 3-group [2,38]. This can be derived from the D5-brane realization of the DWs: the CS action of the D5 is $\mathcal{L}_{C S}=\sum_{p} C_{p} \wedge e^{b_{2}}$. The DWs extend in the $4 \mathrm{~d}$ spacetime such that $F_{4}=\int_{S^{3}} d \mathcal{L}_{C S}=d c_{3}+\frac{M}{2} b_{2}^{2}$, the 3-group follows from the gauge invariance of the worldvolume action of the D5 and is consistent with gauge invariance of (15).

\section{ANOMALIES FROM LITTLE STRING THEORY}

An alternative large $N$ limit of $4 \mathrm{~d} \mathcal{N}=1 \mathrm{SYM}$ is the solution in [39], which is a topologically twisted $S^{2}$ reduction of 6d little string theory (LST) on N NS5-branes in IIB, which in the IR is 6d $(1,1)$ SYM. We now derive the chiral symmetry breaking in $4 \mathrm{~d} \mathcal{N}=1 \mathrm{SYM}$ for gauge group $G$ from a twisted $S^{2}$ reduction of LST. The anomaly polynomial of LSTs has a term [40]

$$
I_{8, \text { mixed }} \supset 2 h_{G}^{\vee} c_{2}\left(R_{6 d}\right) c_{2}\left(F_{G}\right),
$$

where $R_{6 d}, F_{G}$ are the $S U(2)_{R}$ and gauge bundles, respectively. We consider the decomposition $R_{6 d}=(R \otimes$ $\left.K_{S^{2}}^{1 / 2}\right) \oplus\left(R \otimes K_{S^{2}}^{1 / 2}\right)^{\vee}$, where $R$ is the $4 \mathrm{~d} U(1)_{R}$ bundle and $K_{S^{2}}$ is the canonical bundle on $S^{2}$. The twist is realized by $c_{2}(R)=-\left(d A_{R}+\frac{1}{2} c_{1}\left(K_{S^{2}}\right)\right)^{2}$, where $A_{R}$ is associated to the $4 \mathrm{~d} U(1)_{R}$. Integrating (16) on $S^{2}$,

$$
I_{6}=\int_{S^{2}} I_{8, \text { mixed }} \supset 2 h_{G}^{\vee} d A_{R} \wedge c_{2}\left(F_{G}\right) .
$$

This is the ABJ anomaly of $4 \mathrm{~d} \mathcal{N}=1 \mathrm{SYM}$, and it trivializes on a closed 6-manifold, $Y_{6}$, when $2 h_{G}^{\vee} d A_{R}=0$, i.e., when $A_{R}$ is in $\mathbb{Z}_{2 h_{G}^{\vee}}$. In fact, this corresponds to the breaking of the $U(1)_{R} \rightarrow \mathbb{Z}_{2 h_{G}^{\vee}}$ by the ABJ anomaly.

We now also derive the mixed 0-/1-form 't Hooft anomaly between $\mathbb{Z}_{2 h_{G}^{\vee}}$ and 1-form symmetry $\Gamma^{(1)}=Z(G)$, where $Z(G)$ is the center of the simply connected $G$. Let $\mathcal{M}_{5}=\partial Y_{6}$ and consider the anomaly of the $4 \mathrm{~d}$ theory, $I_{5} \supset 2 h_{G}^{\vee} A_{R} \wedge$ $c_{2}\left(F_{G}\right)$ such that $I_{6}=d I_{5}$. Activating a 1 -form symmetry background $b_{2} \in H^{2}\left(\mathcal{M}_{4}, Z(G)\right)$, makes the instanton density fractional, since it is evaluated in $G / Z(G)$ bundles, and $I_{5}$ becomes

$$
I_{5} \supset 2 h_{G}^{\vee} \alpha_{G} A_{R} \cup \mathfrak{P}\left(b_{2}\right),
$$

where $\mathfrak{P}\left(b_{2}\right)$ is the Pontryagin square [41] and for $S U(N) \alpha_{G}=\frac{N-1}{2 N}$. In particular, $A_{R}$ is not a $U(1)_{R}$ bundle, but rather a $\mathbb{Z}_{2 h_{G}^{\vee}}$ surviving subbundle, i.e., nontrivial configuration such that $2 h_{G}^{\vee} d A_{R}=0$ on a closed $Y_{6}$. Note that due to this, the anomaly does not trivialize anymore when $\alpha_{G}$ is fractional $\left(\oint \mathfrak{P}\left(b_{2}\right) \in \mathbb{Z}\right)$. $A_{R}$ has then periodicity $\oint A_{R} \in$ $\frac{\mathbb{Z}}{2 h_{G}^{v}}$ and from (18) we have recovered the full mixed anomaly between the chiral symmetry and the 1-form symmetry, which we derived holographically in the previous section.

\section{ACKNOWLEDGMENTS}

We thank D. Cassani, O. Bergman, L. Bhardwaj, P. Benetti-Genolini, F. Bonetti, C. Closset, M. Hübner, C. Nuñez, and L. Tizzano for discussions and/or comments on the draft. This work is supported by ERC Grant No. 682608. S. S.-N. also acknowledges support through the Simons Foundation Collaboration on "Special Holonomy in Geometry, Analysis, and Physics", Grant No. 724073, Schafer-Nameki.

\section{APPENDIX A: FULL KS SOLUTION, DEFORMED CONIFOLD, AND 5d TOPOLOGICAL COUPLING IN THE IR}

The full KS solution comes with an intricate hierarchy of length scales, which is of central importance for its interpretation as dual to a nonconformal field theory. The starting point is a UV scale at radial distance $r=r_{0}$, where the effective number of D3s $\mathcal{K}\left(r_{0}\right)$ coincides with the actual number $N$ of D3s probing the conifold. As discussed in the main text, the RG flow of the field theory is dual to a stepwise inward flow along the radial direction in steps

$$
r_{k}=r_{0} e^{-\frac{2 \pi k}{3 g_{s} M}},
$$

where $k$ is an integer. We are mainly interested in the case where $N=\kappa M$ for some positive integer $\kappa$, for which the dual field theory flows to $S U(M) S Y M$ in the IR. In this case the effective D3 charge $\mathcal{K}\left(r_{\kappa}\right)=0$ becomes negative past

$$
r_{\kappa}=r_{0} e^{-\frac{2 \pi \kappa}{3 g_{s} M}}=r_{0} e^{-\frac{2 \pi N}{3 g_{s} M^{2}}}
$$

so the UV solution (3) is ill defined in this regime. We also note that the metric (3) has a naked singularity at $r=r_{s}$ with

$$
r_{s}=r_{0} e^{-\frac{2 \pi N}{3 g_{s} M^{2}}-\frac{1}{4}}=r_{\kappa} e^{-\frac{1}{4}}
$$

where the warp factor vanishes $\mathcal{R}\left(r_{s}\right)=0$. However, the UV solution (3) breaks down before this singularity is reached since $r_{\kappa}>r_{s}$. From the above it is clear that the UV and IR regimes are well separated if $\frac{N}{M^{2}} \gg 1$ (implying $\kappa \gg M$ ). The hierarchy of scales can then be summarized as $r_{0} \gg r_{k} \gg r_{k}>r_{s}$, and we can take $r_{s} \rightarrow 0$.

At small radial distances $r \rightarrow r_{s}$, the KS solution is sensitive to the deformation of the conifold induced by the $M$ D5 branes wrapping $S^{2} \subset T^{1,1}$. The nonzero $F_{3}$ flux 
threading the $S^{3}$ prevents this cycle from shrinking to zero volume, whereas the $S^{2}$ collapses. Here the effective number of D3s is zero, and the gauge theory dual is the IR regime of pure $\mathcal{N}=1 S U(M)$ SYM. The warped, deformed conifold is parametrized by a new coordinate $\tau$, which, at large $\tau$, is related to $r$ by $r^{2}=32^{-5 / 3} \epsilon^{4 / 3} e^{2 \tau / 3}$. Near $\tau \rightarrow 0$ the metric is approximately $\mathbb{R}^{3,1}$ times the deformed conifold [17]. We refer to this as the IR KS solution. For the sake of illustration we include the shrinking $S^{2}$ in the degenerate metric

$$
\begin{aligned}
d s_{10}^{2} & =c_{1} \epsilon^{-4 / 3}\left(g_{s} M l_{s}^{2}\right)^{-1} \mathrm{~d} \vec{x}^{2}+c_{2} g_{s} M l_{s}^{2} d s_{6}^{2}, \\
d s_{6}^{2} & =\frac{1}{2} d \tau^{2}+\frac{1}{2}\left(g^{5}\right)^{2}+\left(g^{3}\right)^{2}+\left(g^{4}\right)^{2}+\frac{1}{4} \tau^{2}\left[\left(g^{1}\right)^{2}+\left(g^{2}\right)^{2}\right],
\end{aligned}
$$

where $\left\{g^{i}\right\}$ are the standard bases of 1-forms on $T^{1,1}$ [42], and $c_{i}$ are numerical constants [43]. For $g_{s} M \ll 1$ the curvatures are small everywhere, even in the far IR, such that the supergravity approximation is always reliable. At $\tau=0$ the flux background has significant simplifications,

$$
F_{5}=0, \quad H_{3}=0, \quad F_{3}=\frac{l_{s}^{2} M}{2} g^{5} \wedge g^{3} \wedge g^{4} .
$$

Since the $S^{2}$ shrinks to zero radius as $\tau \rightarrow 0$, we modify our ansatz to ignore any fluctuations over the 2 -sphere. This has the immediate consequence of removing all $F_{5}$ fluctuations, $\delta F_{5}=0$, as we would otherwise be unable to enforce self-duality. The ansatz is therefore $\delta H_{3}=h_{3}, \delta F_{3}=g_{3}$. The Bianchi identities imply that $g_{3}=d c_{2}, h_{3}=d b_{2}$ are closed, and the equations of motion for $\mathrm{H}_{3}$ and $F_{3}$ furnish two kinetic terms,

$$
d_{{ }^{5}} h_{3}=0, \quad d_{{ }^{5}} g_{3}=0 .
$$

Contrary to the UV KS solution, the kinetic terms are not suppressed in this regime. We get a single nontrivial contribution from the $F_{5}$ Bianchi identity implying that

$$
M d b_{2}=0 .
$$

We have no freedom in selecting boundary conditions here; the holographic duality picks out a confining vacuum and, in particular, a global form of the gauge group, namely, the $S U(M)$ simply-connected group, with an unbroken electric 1 -form symmetry $\mathbb{Z}_{M}^{(1)}$. From the bulk perspective, F1s can end on the boundary, indicating the presence of Wilson lines in the gauge theory. Furthermore, $M$ fundamental strings are screened by wrapped D5s that correspond to the "baryon vertex" in the $4 \mathrm{~d}$ theory.

\section{APPENDIX B: EQUATIONS OF MOTION AND 5d SUPERGRAVITY}

In this Appendix we derive the equations of motion of the $5 \mathrm{~d}$ effective theory, obtained from compactifying $10 \mathrm{~d}$ IIB supergravity on $T^{1,1}$. We isolate dominant topological couplings and determine an effective $5 \mathrm{~d}$ action, which governs them. To identify the topological couplings, we expand field strengths $F_{q}$ along $\omega_{p} \in H^{p}\left(T^{1,1}, \mathbb{Z}\right)$ as $F_{q}=\sum_{p} f_{q-p} \wedge \omega_{p}$, and insert these into the Type IIB equations

$$
\begin{aligned}
& d H_{3}=0, \quad d *_{10} H_{3}=-g_{s}^{2} F_{5} \wedge F_{3}, \\
& d F_{3}=0, \quad d_{{ }^{*} 5} F_{3}=F_{5} \wedge H_{3}, \\
& d F_{5}=H_{3} \wedge F_{3}, \quad{ }_{*} F_{5}=F_{5} .
\end{aligned}
$$

The couplings obtained in this way can equally be thought of as embedded into some consistent truncation (e.g., [22]). We find the following topological term in the $5 \mathrm{~d}$ reduction in the UV KS solution,

$$
S_{\text {top }}=2 \pi \int_{\mathcal{M}_{5}} b_{2} \wedge\left(N d c_{2}-M d a_{2}\right) \text {. }
$$

In this section we focus on the UV regime: large $r \sim r_{0}$, dual to the top of the cascade where both cycles $S^{2} \times S^{3} \subset$ $T^{1,1}$ are nondegenerate. We expand the fluctuations along the volume forms $\omega_{2,3} \in H^{*}\left(T^{1,1}, \mathbb{Z}\right.$ ) (see, e.g., [19] for conventions and an explicit parametrization)

$$
\begin{aligned}
& \delta F_{3}=g_{3}+\pi l_{s}^{2} \omega_{2} \wedge g_{1}, \quad \delta H_{3}=h_{3}, \\
& \delta F_{5}=\pi l_{s}^{2} \omega_{2} \wedge f_{3}+\frac{2 \pi l_{s}^{2}}{6} \mathcal{R} \omega_{3} \wedge * f_{3} .
\end{aligned}
$$

Here, $h_{3}, g_{1,3}, f_{3}$ are all external fields, and in this Appendix we restore prefactors for completeness. Selfduality of $\delta F_{5}$ implies a choice of frame: we can fix one expansion component in terms of the other. We use the 3 -form piece, since the operators of the boundary 1-form symmetry are manifest in this frame. The Bianchi identities for $H_{3}, F_{3}$ imply that the corresponding $5 \mathrm{~d}$ fields are closed, so we write $h_{3}=d b_{2}, g_{3}=d c_{2}, g_{1}=d c_{0}$. We interpret $c_{0}$ as an axion, whereas $b_{2}, c_{2}$ couple to F1s and D1s, respectively. The Bianchi identity for $\delta F_{5}$ implies that $f_{3}$ is not closed,

$$
d f_{3}=\mathrm{d} \mathcal{L} \wedge g_{3}+h_{3} \wedge g_{1} .
$$

As such, we shift the field to obtain closure and define a new gauge potential $d a_{2}=f_{3}-\mathcal{L} d c_{2}-b_{2} d c_{0}$, which couples to D3s wrapping $S^{2} \subset T^{1,1}$. The 5 d equations of motion are 


$$
\begin{aligned}
d\left(\mathcal{R}_{{ }} f_{3}\right) & =\frac{3}{2 \pi} M d b_{2}, \\
d\left(\mathcal{R}^{5}{ }_{{ }_{5}} d b_{2}\right) & =-27 \pi l_{s}^{4} g_{s}^{2}\left(\mathcal{K} d c_{2}-M f_{3}+\frac{2 \pi}{3} \mathcal{R}_{{ }_{5}} f_{3} \wedge d c_{0}\right), \\
d\left(\mathcal{R}^{5}{ }_{{ }_{5}} d c_{2}\right) & =27 \pi l_{s}^{4}\left(\mathcal{K} d b_{2}+\frac{2 \pi}{3} \mathcal{R}_{*_{5}} f_{3} \wedge \mathrm{d} \mathcal{L}\right), \\
d\left(\mathcal{R}_{*_{5}} d c_{0}\right) & =-\mathcal{R}_{*_{5}} f_{3} \wedge d b_{2} .
\end{aligned}
$$

We are interested in studying the theory near a slice defined by $r=r_{k}$ for some $k \ll \frac{N}{M}$, i.e., a slice which is far away from the IR, where the UV KS solution is valid. Parametrizing the radial coordinate as $r=r_{k}+r^{\prime}$ where $r^{\prime} \ll r_{k}$ we find that the effective D3 charge is $\mathcal{K}(r)=N-k M+\mathcal{O}\left(r^{\prime} / r_{k}\right)$. At long distances (near $r_{k}$ with $k \ll \frac{N}{M}$ ) the kinetic terms and couplings of higher mass dimension will be suppressed with respect to terms with fewer derivatives. From these equations of motion we extract leading topological contributions,

$$
N d b_{2}=0, \quad M d b_{2}=0, \quad N d c_{2}-M d a_{2}=0,
$$

where we ignore $c_{0}$, which can be gauged away via a Stückelberg mechanism. From these we construct the action (8).

\section{APPENDIX C: 5d CONSISTENT TRUNCATION}

An important component of the analysis presented in this paper is the consistent truncation of IIB supergravity to $5 \mathrm{~d}$ for conifold solutions. In [22] such a consistent truncation was found which encompasses both the UV and IR KS solutions, and where we show the holographic realization of the ABJ anomaly and the mixed 0-/1-form symmetry anomaly are both manifest. In what proceeds, we present the map required to translate between our work and their notation. The KS flux background is parametrized as

$F_{3}=q \Phi \wedge \eta, \quad B_{2}=b^{\Phi} \Phi, \quad F_{5}=-\left(k-q b^{\Phi}\right) \Phi \wedge \Phi \wedge \eta$,

where $\Phi, \eta$ are left-invariant forms on $T^{1,1}$. They are related to the volume forms of $S^{2}$ and $S^{3}$ as $\Phi=\frac{1}{3} \omega_{2}$, $\Phi \wedge \eta=-\frac{1}{9} \omega_{3}$. We rescale the IIB fields by

$F_{3} \rightarrow-\frac{9 l_{s}^{2}}{2} F_{3}, \quad B_{2} \rightarrow-3 \pi l_{s}^{2} B_{2}, \quad F_{5} \rightarrow \frac{27 \pi l_{2}^{4}}{2} F_{5}$,

which ensures that the background is quantized as

$$
\int_{S^{3}} \frac{F_{3}}{\left(2 \pi l_{S}\right)^{2}}=q \in \mathbb{Z}, \quad \int_{T^{1,1}} \frac{F_{5}}{\left(2 \pi l_{S}\right)^{4}}=k \in \mathbb{Z},
$$

and furthermore gives the identifications

$$
q=M, \quad b^{\Phi}=-\mathcal{L}, \quad k=N .
$$

Notice that the rescalings are consistent: they give rise to the same factor on either side of the Bianchi identity for $F_{5}$. These normalizations also imply that we should identify the fluctuation $d c^{\Phi}=-\frac{2 \pi}{3} d c_{0}$. Finally, we rescale the $U(1)$ gauge field in [22] $A \rightarrow \frac{4 \pi}{3} A$ so that it is normalized as in [44]. The 5d topological couplings obtained in [22] are

$\mathcal{L}_{5 d}=\mathcal{R}\left|g_{1}^{\Phi}\right|^{2}-\frac{1}{2} f_{2}^{\Phi} \wedge\left(-q b_{2} \wedge A+b_{2} \wedge D c^{\Phi}\right)$,

$f_{2}^{\Phi} \supset q b_{2}, \quad g_{1}^{\Phi} \supset D c^{\Phi}, \quad D c^{\Phi}=d c^{\Phi}-q A$.

Using the map detailed above gives the action (11).

\section{APPENDIX D: ALTERNATIVE BOUNDARY CONDITIONS}

We derived a UV topological action (8) after dimensional reduction of the KS solution at fixed $r$ slices. For this action we considered one choice of boundary conditions, $b_{2}$ is Dirichlet and $\mathcal{C}$ Neumann. In this Appendix, we provide a comprehensive analysis of all b.c.s. Clearly an alternative is to exchange the two gauge fields and consider

$$
b_{2} \text { Neumann, } \quad \mathcal{C} \text { Dirichlet. }
$$

Now the topological operator $U_{c}(\partial \Sigma)=e^{2 \pi i \oint_{\partial \Sigma} \mathcal{C}}$ on $\partial \Sigma \subset$ $\left.\mathcal{M}_{5}\right|_{r_{k}}$ gives rise to the charged operators of the field theory, whereas $U_{b}\left(M_{2}\right)$ for closed $M_{2}$ with $\partial M_{2}=\emptyset$ defines the charge operators, and we again find the 1-form symmetry (5). Physically, this choice of boundary conditions means that bound states of D1 strings and D3 branes wrapped on $S^{2}$ can end on the boundary to form line operators. From the Bianchi identities we find that $\operatorname{gcd}(N, M)$ many of the D1-D3 bound states can end on an NS5 brane wrapping $T^{1,1}$. This provides a screening mechanism for the line operators and is consistent with the 1-form symmetry. This configuration of lines corresponds in the dual field theory to the 't Hooft lines of the SYM theory.

We can consider general boundary conditions on the fields through gauging. Let $\operatorname{gcd}(N, M)=p q$. We can then consider the theory,

$S_{\mathrm{CS}^{\prime}}=\frac{1}{2 \pi} \int\left(p b_{2}^{\prime} \wedge d \mathcal{C}^{\prime}+q b_{2}^{\prime \prime} \wedge d \mathcal{C}^{\prime \prime}+b_{2}^{\prime \prime} \wedge d \mathcal{C}^{\prime}\right)$

We can recover the action (8) by integrating out $b_{2}^{\prime \prime}$ and $\mathcal{C}^{\prime}$. Writing it in this way, we see that these backgrounds can be coupled to a $4 d$ theory in a way that exhibits $\mathbb{Z}_{p} \times \mathbb{Z}_{q}$ 1 -form symmetry, where the line operators are realized in terms of $(p, q)$-strings, where the D-strings also include wrapped D3-branes. 
From a bulk point of view we can see the screening by considering $(p, q) 5$-branes wrapping $T^{1,1}$. The Bianchi identities for $(p, q)$ 5-branes are

$$
p \int_{T^{1,1}} d F_{7}=N H_{3}, \quad \frac{q}{\left(2 \pi l_{s}\right)^{4}} \int_{T^{1,1}} d H_{7}=\frac{q}{\left(2 \pi l_{s}\right)^{4}} \int_{T^{1,1}} F_{3} \wedge F_{5}=(N-k M) F_{3}-M d a_{2} .
$$

Therefore bound states of $q$ F1-strings and $p$ D1-D3 bound states can end on a $(p, q) 5$-brane. This process is dual to the baryon vertex screening the line operators of the dual theory, which are given by the bound states of $q \mathrm{~F} 1$-strings and $p$ D1-D3, at constant $r_{k}$ slices.

The existence of alternative b.c.s will potentially lead in the IR to the strongly coupled vacua of $\mathcal{N}=1 P S U(M)$
SYM, when $N$ is a multiple of $M$. Since one of the vacua has a different (deconfining) behavior, we believe that this provides evidence for the existence of a new holographic solution in the IR describing this deconfining vacuum, the spontaneous breaking of the 1-form symmetry, and therefore this phase transition.
[1] O. Aharony, N. Seiberg, and Y. Tachikawa, J. High Energy Phys. 08 (2013) 115.

[2] D. Gaiotto, A. Kapustin, N. Seiberg, and B. Willett, J. High Energy Phys. 02 (2015) 172.

[3] E. Witten, Nucl. Phys. B202, 253 (1982).

[4] C. Córdova and T. T. Dumitrescu, arXiv:1806.09592.

[5] C. Cordova and K. Ohmori, arXiv:1910.04962.

[6] E. Witten, J. High Energy Phys. 12 (1998) 012.

[7] O. Aharony and E. Witten, J. High Energy Phys. 11 (1998) 018.

[8] D. J. Gross and H. Ooguri, Phys. Rev. D 58, 106002 (1998).

[9] D. M. Hofman and N. Iqbal, SciPost Phys. 4, 005 (2018).

[10] O. Bergman, Y. Tachikawa, and G. Zafrir, J. High Energy Phys. 07 (2020) 077.

[11] I. Bah, F. Bonetti, and R. Minasian, J. High Energy Phys. 03 (2021) 196.

[12] N. Seiberg and E. Witten, Nucl. Phys. B426, 19 (1994); B430, 485(E) (1994).

[13] N. Seiberg and E. Witten, Nucl. Phys. B431, 484 (1994).

[14] A. Klemm, W. Lerche, S. Yankielowicz, and S. Theisen, Phys. Lett. B 344, 169 (1995).

[15] P. C. Argyres and A. E. Faraggi, Phys. Rev. Lett. 74, 3931 (1995).

[16] I. R. Klebanov and N. A. Nekrasov, Nucl. Phys. B574, 263 (2000).

[17] I. R. Klebanov and M. J. Strassler, J. High Energy Phys. 08 (2000) 052.

[18] I. R. Klebanov and A. A. Tseytlin, Nucl. Phys. B578, 123 (2000).

[19] C. P. Herzog, I. R. Klebanov, and P. Ouyang, arXiv:hep-th/ 0108101.

[20] I. R. Klebanov, P. Ouyang, and E. Witten, Phys. Rev. D 65, 105007 (2002).

[21] M. J. Strassler, arXiv:hep-th/0505153.

[22] D. Cassani and A. F. Faedo, Nucl. Phys. B843, 455 (2011).
[23] I. Bena, G. Giecold, M. Grana, N. Halmagyi, and F. Orsi, J. High Energy Phys. 04 (2011) 021.

[24] J. Schon and M. Weidner, J. High Energy Phys. 05 (2006) 034.

[25] M. Atiyah, J. M. Maldacena, and C. Vafa, J. Math. Phys. (N.Y.) 42, 3209 (2001).

[26] See Supplemental Material at http://link.aps.org/ supplemental/10.1103/PhysRevD.104.066005 for Details on the supergravity.

[27] N. Seiberg, Nucl. Phys. B435, 129 (1995).

[28] A. Dymarsky, I. R. Klebanov, and N. Seiberg, J. High Energy Phys. 01 (2006) 155.

[29] F. Benini, F. Canoura, S. Cremonesi, C. Nunez, and A. V. Ramallo, J. High Energy Phys. 09 (2007) 109.

[30] D. Belov and G. W. Moore, arXiv:hep-th/0412167.

[31] J. M. Maldacena, G. W. Moore, and N. Seiberg, J. High Energy Phys. 10 (2001) 005.

[32] The operators $U(M)$ are defined in the same way for spaces with boundary, and we will use the same notation for the restriction of these operators to the boundary of spacetimethis should be unambiguous from the context.

[33] E. Witten, J. High Energy Phys. 07 (1998) 006.

[34] I. e. $b_{2} \in H^{2}\left(\mathcal{M}_{5}, \mathbb{Z}_{M}\right)$ and $A \in H^{1}\left(\mathcal{M}_{5}, \mathbb{Z}_{2 M}\right)$, and using the cup-product instead of wedge.

[35] D. Gaiotto, Z. Komargodski, and N. Seiberg, J. High Energy Phys. 01 (2018) 110.

[36] M. Berg, M. Haack, and W. Mueck, Nucl. Phys. B736, 82 (2006).

[37] G. Papadopoulos and A. A. Tseytlin, Classical Quantum Gravity 18, 1333 (2001).

[38] N. Seiberg, Y. Tachikawa, and K. Yonekura, Prog. Theor. Exp. Phys. 2018, 073 B04 (2018).

[39] J. M. Maldacena and C. Nunez, Phys. Rev. Lett. 86, 588 (2001).

[40] C. Cordova, T. T. Dumitrescu, and K. Intriligator, J. High Energy Phys. 04 (2021) 252. 
[41] C. Córdova, D. S. Freed, H. T. Lam, and N. Seiberg, SciPost Phys. 8, 002 (2020).

[42] R. Minasian and D. Tsimpis, Nucl. Phys. B572, 499 (2000).

[43] I. R. Klebanov and E. Witten, Nucl. Phys. B536, 199 (1998).
[44] C. P. Herzog, I. R. Klebanov, and P. Ouyang, in Les Houches Summer School: Session 76: Euro Summer School on Unity of Fundamental Physics: Gravity, Gauge Theory and Strings (Springer Science \& Business Media, Berlin, 2002), pp. 189-223. 